\title{
LASER LIGHT CONTROL OF SELF-ORGANIZATION PROCESS
}

\author{
YUKIMASA MATSUMURA \\ Graduate School of Science and Technology, Shizuoka University, 3-5-1 Johoku \\ Hamamatsu, Shizuoka 432-8561, Japan \\ f5945008@ipc.shizuoka.ac.jp \\ WATARU INAMI \\ Division of Global Research Leaders, Shizuoka University, 3-5-1 Johoku \\ Hamamatsu, Shizuoka 432-8561, Japan \\ Japan Science and Technology Agency, CREST \\ inami@eng.shizuoka.ac.jp \\ YOSHIMASA KAWATA \\ Graduate School of Science and Technology, Shizuoka University \\ Japan Science and Technology Agency, CREST \\ kawata@eng.shizuoka.ac.jp \\ Received (Day Month Year)
}

\begin{abstract}
We present a controlling technique of microporous structure by laser irradiation during self-organization process. Polymer solution was dropped on the substrate at high humid condition and the honeycomb structure of regularly aligned pores on the film was fabricated by attaching of water droplets on the solution surface. We demonstrated that it was possible to prevent forming of pores at the region of laser irradiation and flat surface was fabricated. The method of our study is microfabrication processing technique that combines the advantages of bottom up and top down techniques. This method is expected that it is applied to photonic crystals, biological cell culturing, surface science and electronics fields.
\end{abstract}

Keywords: laser processing; microfabrication; self-organization; microporous; honeycomb film; laser irradiation.

\section{Introduction}

Microfabrication techniques have been developed by many researchers with the demand of nanotechnologies. ${ }^{1}$ Microfabrication techniques are categorized as top down or bottom up fabrication techniques. One of typical top down methods is photolithography. The advantages of the top down methods are high precision and high reproducibilities in the fabrication process. ${ }^{2}$ The top down method, however, requires expensive equipments, long processing time, and complicated procedures.

On the other hand, bottom up techniques are known as self-organization process. The bottom up methods have the advantages of short process time and low cost. It is easy to produce a uniform pattern in large area, while, the bottom up techniques are difficult to produce arbitrary structures. ${ }^{3}$ 
We propose controlling of self-organization porcesses by laser irradiation in order to produce arbitrary structures. It has been reported that microporous films are formed by the cast on the glass substrate under the high humid condition. ${ }^{4-11}$ The technique has a potential to be applied many applications, such as photonic crystals, cell culturing, water repellent materials, field emitters, etc. ${ }^{12-16}$ We present that the microporous structures can be controlled by the irradiation of pulsed laser during self-organized process.

\section{Laser Control of Self-organized Process}

\subsection{Fabrication of Self-organized Microporous Films}

It has been reported that the fabrication of honeycomb microporous films are fabricated by self-organization process. ${ }^{4}$ The principle of fabrication process of honeycomb structure on a film is explained as follows.

Polymers with hydrophilic group are dissolved in highly volatile solvent. This solution is dropped on a glass substrate in high humid air. Air near the surface of the solution is cooled because the solvent draw heat during evaporation. As a result of it, water droplets are generated near the solution surface and they attach on the surface. Capillary force is operated toward three-pahse line of the solution and water droplets on the solution surface are packed as honeycomb structure by capillary force. Finally, the solvent is completely evaporated and micropores are produced at the position of water droplets are attached on the solution surface.

We used a solution of polystyrene, monocarboxy terminated (Mw: 50,000, Scientific Polymer Products, Inc.) in chloroform (Kanto chemical co., Inc.). This polymer has carboxyl group as hydrophilic group.

The polystyrene chloroform solution of $25-50 \mu \mathrm{L}$ is dropped onto glass substrate at high humid condition of $75 \%$ r.h. The fabricated microporous films are observed with a scanning electron microscope (SEM; JSM-6390, JEOL).

\subsection{Laser Control of Self-organization Process}

Figure 1 shows the optical setup to control of self-organization process by laser irradiation during fabrication of microporous film. A Q-switched Nd: YAG laser (wavelength: $532 \mathrm{~nm}$, pulse width: $3-5 \mathrm{~ns}$, repetition frequency: $1 \mathrm{~Hz}$ ) is used as a light source. Laser light is focused by an objective lens on the solution surface. The magnification and numerical aperture of the objective lens are 20x and 0.4, respectively. We irradiated only one pulse of $20 \mu \mathrm{J}$. Since the position of solution surface is moved during evaporation, we tracked the solution surface with moving the glass substrate in the axial direction. 


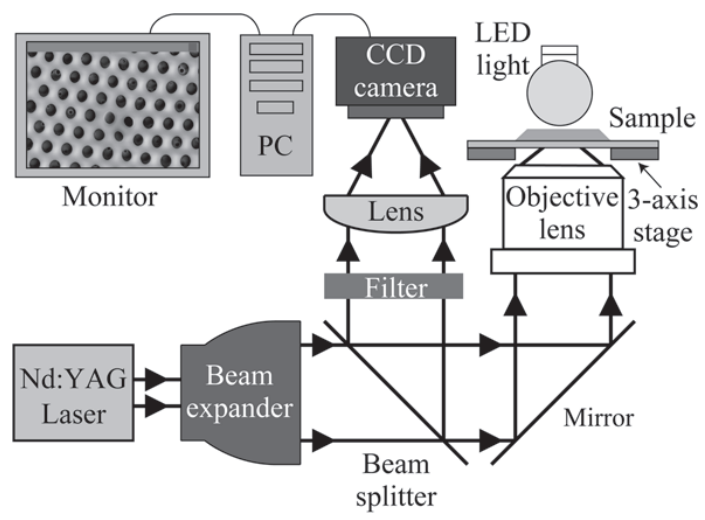

Fig. 1. Self-organization control optical setup.

\section{Experimental Demonstration of Laser Control}

The surface of solution droplet (solution concentration: $40 \mathrm{~g} / \mathrm{L}$, cast volume: $25 \mu \mathrm{L}$ ) is irradiated by the laser after 53 seconds from solution drop on the cover glass. Figure 2 shows SEM images of self-organization microporous polystyrene film with irradiation of a laser pulse. Figure 2(a) shows entire image of laser irradiated area and Fig. 2(b) shows magnified image near the boundary of microporous area and flat area produced by laser irradiation. We can recognize clearly plane suraface without pores area was produced by laser irradiation. The result shows that we can prevent forming pores at laser irradiated area. As a result of it, we may say self-organization process can be controlled by a pattern of laser irradiation.
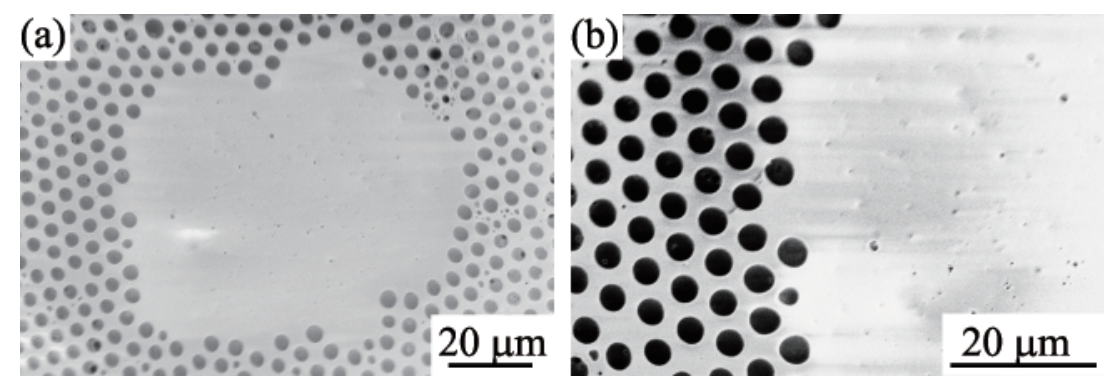

Fig. 2. SEM images of self-organization control microporous polystyrene film by laser irradiation (a) entire picutre and (b) magnified picture.

The combination structure of microporous and plane surface can be produced by laser irradiation during formation of micropores with self-organization process. When laser is irradiated after self-organization process are done, laser ablation of 
the film should be required and it is not possible to get the structure shown in Fig. 2(b). For laser ablation process higher laser pulse energy is required.

Figure 3 shows mechanism of controlling self-organization process by laser irradiation. We believe that water droplets on the solution surface are removed by shock wave that generated by laser irradiation. Because the solution droplet is irradiated by a laser pulse just before it is completely evaporated, there are no enough time that water droplets are attached in this area again and the plane surface without pores is generated.

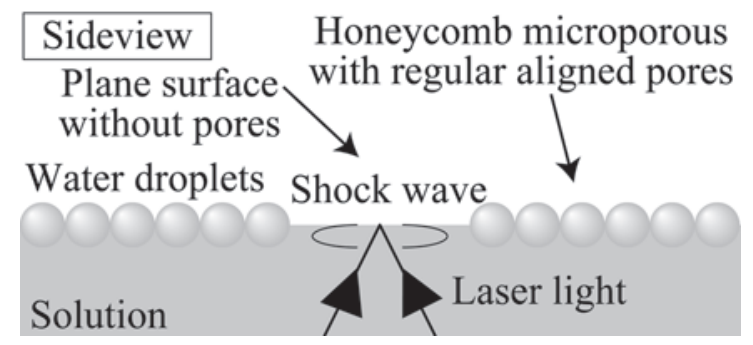

Fig. 3. Side view pattern diagram of solution surface with laser irradiation.

We tried to control the size of area induced with laser irradiation by changing the solution viscosity and capillary force. The solution of concentration solution(solution concentration: $50 \mathrm{~g} / \mathrm{L}$, cast volume: $50 \mu \mathrm{L}$ ) is dropped on the cover glass and the concentration of solution was controlled by evaporation time, namely time interval between solution drop and laser irradiation. A laser pulse is irradiated at 75, 79 and 83 seconds after solution was casted on the cover glass. When laser light is irradiated with the shorter interval than 75 seconds, the area of laser irradiation is totally coverd with water droplets again by capillary force and no flat surface is produced. For longer interval than 83 seconds, polystyrene microporous film is damaged by laser light due to high viscosity of the solution.

Figure 4 shows SEM images of laser controlled area with the intervals of (a) 75 seconds, (b) 79 seconds, and (c) 83 seconds after solution was casted on a cover glass. The flat areas are $1150,2500,7200 \mu \mathrm{m}^{2}$ for the intervals of 75,79 , and 83 seconds, respectively. The larger intervals result the larger flat areas, because the viscosity of the solution becomes higher and water droplets can not cover the area again. The shape of the laser controlled area shown in Fig. 2 is close to circular, but the shapes in Fig 4 are not circular. We believe that that the shape of the controlled area was determined by the balance of shock wave that removed the water droplets and capillary force to pack the water droplets as honeycomb structure. The capillary force strongly depends on the position of laser irradiation. At the center of the solution, capillary force is almost same in the all direction, but at the edge of the solution it is not symmetry. In Fig. 2, we irradiated laser at the center, but in Fig. 4 
we irradiated laser on the side of the solution. We believe that asymmetric capillary force was main reasons for the shapes of controlled area shown in Fig. 4.

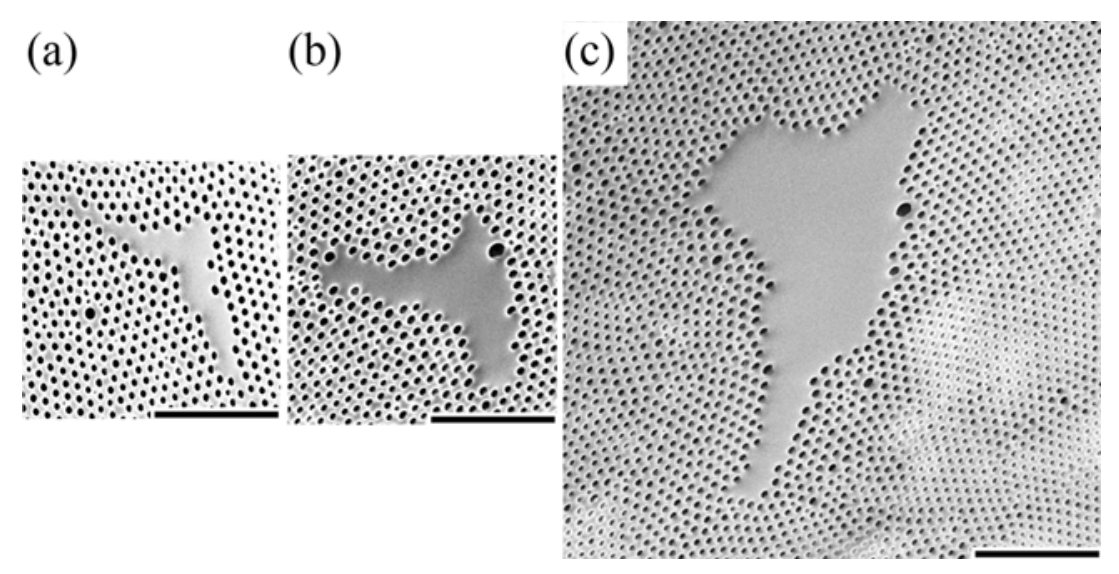

Fig. 4. SEM images of laser controlled area with the intervals of (a) $75 \mathrm{~s}$, (b) $79 \mathrm{~s}$, and (c) $83 \mathrm{~s}$ after solution casting on a cover glass, respectively. Scale bar: $50 \mu \mathrm{m}$.

As a conclusion of this preliminary experiments, we may say it is possible to control self-organization process and area of affected region by changing the time interval of laser pulse irradiation after solution is casted.

We also tried to fabricate a line structure by multiple irradiation of laser pulses. Three shots of laser pulse are irradiated and sample on the stage is moved with the rate of $100 \mu \mathrm{m} / \mathrm{s}$. Three laser pulses are irradiated 75 seconds after solution was casted on the cover glass.

Figure 5 shows SEM image of line pattern fabricated by laser irradiation of three pulses with the movement of sample. The three circles with broken lines indicate the positions that laser pulses are irradiated. A line structure was produced, although the line was curved due to the capillary force. The width of the line area was about $80 \mu \mathrm{m}$. It is possible to fabricate the narrow line structure by sequential laser pulses irradiation and to produce an arbitrary structure by modifing laser irradiation condition.

\section{Conclusion}

We demonstrated that the microporous structure was able to be controlled by laser light irradiation during self-organization process. The plane surface area without pores was obtanined by a shock wave induced with a laser pulse. The area size without pores is also controlled by changing the interval of laser light irradiation from the solution dropped on substrate. A line pattern is fabricated by sequential three laser pulses irradiation. Our method has an potential that combines the processes 


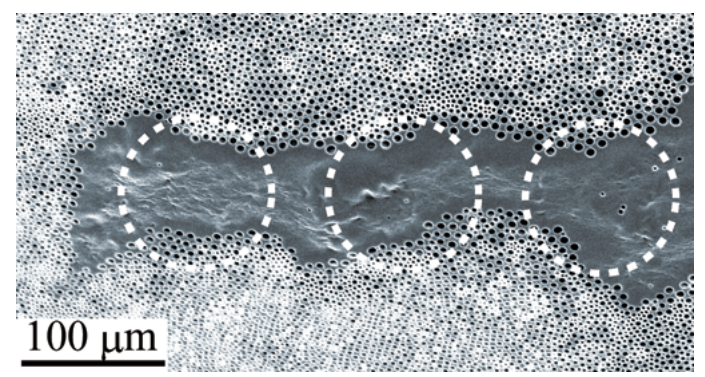

Fig. 5. SEM image of controlled area of line pattern by three shots laser irradiation and sample moving $(100 \mu \mathrm{m} / \mathrm{s})$.

of bottom up and top down methods. It is a promising technique can be applied to produce photonic crystals, biological culturing process, water repellent materials, electronics and so on.

\section{References}

1. M. Madou, Fundamental of Microfabrication, CRC Press LLC (1997).

2. T. Kawai, Graphic Explanation All of Application Artifice of Nanotechnology, Kogyochosakai (2002).

3. Q. Yuan, L. Wan, H. Jude and P. J. Stang, Journal of the American Chemical Society, 127 (46) (2005) 16279.

4. G. Widawski, M. Rawiso and B. Francois, Nature, 369 (1994) 387.

5. N. Maruyama, T. Koito, J. Nishida, T. Sawadaishi, X. Cieren, K. Ijiro, O, Karthaus and M. Shimomura, Thin Solid Films, 327-329 (1998) 854.

6. B. de Boer, U. Stalmach, H. Nijland and G. Hadziioannou, Advanced Materials, 12 (2000) 1581

7. M. H. Stenzel-Rosenbaum, T. P. Davis, A. G. Fane and V. Chen, Angewandte Chemie, 113 (2001) 3536.

8. M. Srinivasarao, D. Collings, A. Philips and S. Patel, Science, 292 (2001) 79.

9. L. Song, R. K. Bly, J. N. Wilson, S. Bakbak, J. O. Park, M. Srinvasarao and U. H. F. Bunz, Advanced Materials, 16 (2004) 115.

10. L. V. Govor, I. A. Bashmakov, F. N. Kaputski, M. Pientka and J. Parisi, Macromolecular Chemistry and Physics, 201 (2000) 2721.

11. Y. Tian, S. Liu, H. Ding, L. Wang, B. Liu and Y. Shi, Polymer, 48 (2007) 2338.

12. J. Ping, H. Xiao-Yong, Y. Hong and G. Qi-Huang, Chinese Physical Letters, Vol. 23, No. 7 (2006) 1813.

13. Y. Nishijima, K. Ueno, S. Juodkazis, V. Mizeikis, H. Misawa, M. Maeda and M. Minaki, Optics Express, Vol. 16, No, 18 (2008) 13676.

14. A. Tsuruma, M. Tanaka, N. Fukushima and M. Shimomura, e-Journal of Surface Science and Nanotechnology, 3 (2005) 159.

15. H. Yabu, M. Tanaka, N. Fukushima and M. Shimomura, Langmuir, 21 (8) (2005) 3235.

16. Y. Hirai, H. Yabu, M. Shimomura, Colloids and Surfaces A: Physicochemical and Engineering Aspects, 313-314 (2008) 312. 